\title{
5 Techniques, Chaîne Opératoire and Technology
}

Most of the phases of the chaîne operatoire involved in pottery production are addressed through the observation and characterization of ceramic fabrics. However, before approaching each of these phases, it is necessary to continue discussing briefly some basic concepts that are necessary to understand the archaeological relevance that has the identification of the materials and techniques related to pottery manufacture. The aim is to highlight the importance that techniques, chaines opératoires and technology have in the study of the archaeological ceramics. All these concepts are closely interrelated and also connected with society. However, they imply different levels of analysis and, therefore, require some clarifications and qualifications.

As noted by Sigaut (1994), the techniques and the technical sequences that we observe in the archaeological record are part of a broader framework, technology. In the study of technology the concept of chaîne opératoire is used to organise the techniques involved in the production and ultimately aims to summarize consistently the technological features used by the individuals from a given society. Meanwhile, as we will see later, it is actually the study of the technology that allows us to approach society, becoming a key issue in archaeology. In short, techniques, chaîne opératoire and technology are concepts closely related, but it is suggested to separate them to properly explain the role that each one plays.

\subsection{Techniques and Technological Choices}

Before dealing with the concept of chaîne operatoire and the role of technology in pottery production we must pay attention to the meaning given to techniques. Techniques are, like the alphabet, the first main level ${ }^{13}$ of encoding technological processes and become one of the key parameters that allow, in short, the development of technological behaviours. The different ways of understanding the techniques have been also applied in the studies of ceramic technology, where it is considered that techniques mean a link between people, crafts and materiality. Thus, we can summarize that techniques are a set of procedures engaged in craftworks and used by people in the process of making objects from an array of materials. In this process the different individuals participate in pottery production in a more or less coordinated way and according to their skill and knowledge in order to fulfil a number of needs (García Rosselló, 2008; Pfaffenberger, 1992).

13 In this sense, it should be considered that there are other secondary elements below techniques, such as the technical gestures or the motor habits. 
The introduction of the concept of technological choice (Lemonnier, 1986) in archaeological studies enabled to develop new viewpoints about the significance of the techniques related to ancient artefacts. From a materialistic sense, we should consider that the techniques and materials used in the manufacture of prehistoric ceramics with the aim to satisfy particular needs are also related to potters' choices. Through these choices, potters determine the formal properties and attributes of the artefacts they produce. Thus, each technological choice made by the craftspeople has an impact on one or more formal properties of the final product. It is by means of these particular choices made among multiple alternatives that the artisans are able to manufacture a specific kind of vessel.

Each human group usually has several possibilities when making a pottery that depends, as discussed below, on a number of factors. Then, humans must choose among different options and this action implies a reflection in which individuals, for certain reasons, either conscious or unconscious, do things in one way and not another. Questioning the choices of certain techniques and materials, their possible causes and consequences and their role in society, we can address aspects of pottery production that go beyond the objects themselves. It is in this framework where ceramic paste analysis may contribute substantially in increasing our knowledge, since it allows approaching the technological choices made by other societies in the past.

In short, we can highlight that technical gestures go further than the mere material aspect and constitute a social fact. Techniques are part of society and, therefore, belong to the same reality. Thus, all technological analysis should start from the premise that we have to approach first the techniques used by individuals in order to address society (Cresswell, 1983, 2003). So, if we characterise in deep the techniques used in the past we will be better positioned to carry out a more complex view of the technology and, by extension, of the social reality (Sigaut, 1994).

\subsection{Introduction to the Chaîne Opératoire}

The concept of chaîne opératoire ${ }^{14}$ entails a proper framework for rebuilding and organising all the technological choices regarding the materials and techniques as well as the operations involved in the pottery production process (Lemonnier, 1976; Sillar and Tite, 2000). Each technique that the potter uses constitutes a minor technological process (Calvo et al., 2004a; García Rossello, 2008). However, the manufacture of pottery comprises a set of techniques and materials that go beyond the individual or isolated technical gestures. Thus, the concept of chaîne opératoire

14 The notion of chaine opératoire, its development in the study of technology and broad literature about this concept can be found in García Rosselló and Calvo (2013). 
encompasses all the techniques and materials used in the manufacture of pottery, overcoming the mere addition of minor technical processes. Moreover, this concept involves the use of recipes related to specific social dynamics that regulate the whole production process, from the raw materials selected to the final product obtained. These recipes are the consequence of the technological knowledge acquired by an individual as a result of her or his social behaviour and interaction. In this sense, the chaîne opératoire consists in socially meaningful operational sequences in which different actions involving specific materials, spaces and tools take place. In these operational sequences even the problems that arise during the manufacturing process are solved in a specific way depending on social consensus.

The notion of chaîne opératoire has been widely used in technological analysis and particularly applied in the study of pottery technology from the 1970s, with significant contributions, among others, from the school of Techniques et Culture of the French CNRS (Balfet, 1965, 1973, 1975, 1991; Cresswell, 1976) and the Department of Pottery Technology of the University of Leiden (Van As, 1984, 1987; Van der Leeuw, 1976a, 1976b, 1993). In recent decades, the application of this concept in the study of ceramics has been systematized and generalized (Gelbert, 2000; Gosselain, 1992a, 2002; Livingstone-Smith, 2007; Roux, 1994).

As will be discussed later, in general, the first approaches developed based on the concept of chaîne opératoire understood the physical process of manufacturing an object as the mere acquisition of skills in terms of technical complexity and technological progress. In these pioneering studies, technology was seen in an evolutionary sense or as closely adapted to the environment in economic and functionalist terms (Prieto, 1999; Sigaut, 1994). In any case, in spite of the limitations of these processual theoretical trends from a current viewpoint, we must recognize that they were the first ones to be aware of the significance of technology in ceramic studies (Matson, 1965a; Rye, 1976, 1981; Shepard, 1971). Thus, these perspectives permitted us to overcome the strict use of typological studies in our discipline around the 1960s (Albero et al., forthcoming).

In the 1980s new viewpoints on technology from anthropology, sociology, as well as from structuralist theories in general were introduced. As a result, technology was linked to social and symbolic phenomena that are characteristic of the societies that perform the technical gestures. It is in these frameworks where concept of chaîne opératoire acquires a greater theoretical and methodological potential to interpret the social phenomena behind the technology of artefacts (Cresswell, 2003; Edmonds, 1990; Lemonnier, 1986, 1990; Martinón-Torres, 2002; Sillar and Tite, 2000; Vidal and García Rosselló, 2009). As J. García Rosselló pointed out (2008), currently the concept of chaîne opératoire entails a procedure that allows identifying the technical processes applied by individuals with the aim to comprehend a given material culture. Thus, the concept has been widely used in the research undertaken in recent decades, becoming a viable theoretical and methodological framework for studying ceramic technology from different schools of thought. 
It actually seems possible to characterize properly the ceramic production process in detail. On the one hand, the increase of experimental and ethnographic research projects has allowed a better understanding and articulation of the technical parameters and social phenomena involved in the different stages of the manufacture process. On the other, the accurate study of this process is also related to the significant improvements undertaken in the methodologies used to study pottery and a more widespread application of archaeometric analyses. In this sense, archaeometric analysis provides accurate information about clay procurement, paste preparation and firing procedures, among other aspects of pottery production. Thus, paste and fabric analyses are particularly useful because they provide reliable data on the technological recipes used by the potters as well as regarding their savoir faire. At this state, the conclusions obtained, once summarized in particular chaînes opératoires, exceed the material dimension of the objects.

Nevertheless, it must be highlighted that the concept of chaîne opératoire is just an analytical process based on researchers' constructions that focuses on human actions through the study of material culture. Therefore, in many cases, the past societies that produced the ceramics could conceive their actions, technological traditions and technical gestures on explanatory basis completely different to the current scientific standards (Vidal and García Rosselló, 2009).

Despite this fact, the study of the chaîne operatoire provides us with certain information about particular sequences of interconnected human actions. So that, the more complete these sequences are, the more precise technological information about people we will have (Schiffer, 2004). In this sense, the chaîne opératoire represents an adequate procedure for an orderly description of ceramic features and a proper evaluation of these operational sequences. Thus, we can investigate the way past actions were interrelated, their variability degree and their frequency, as well as if certain actions were related to specific artefacts or particular contexts (Prieto, 1999; Sillar and Tite, 2000).

In short, the chaîne opératoire is the preferred method for approaching many aspects such as the organization of pottery production, technological change, knowledge transmission and the properties of end products. In this sense, the study of materials, techniques and operational sequences may also allow us to understand some material parameters linked to the pottery production process, such as the ease of manufacture and the durability of ceramics (Schiffer and Skibo, 1987; Van As, 1984). The chaîne opératoire is an adequate strategy to order and compare the multiple technical choices made by the potters as well as a coherent framework to establish the relationships existing between the materials and techniques involved in the entire pottery production process. Finally, it should be noted that this concept also entails chronological sequences in relation to successive technical actions that occur at certain times as well as specific places that involve a particular materiality such as architectonic structures, tools, etc. 


\subsection{Technology and Ceramics}

This theoretical and methodological development regarding techniques was similarity reflected in the concept of technology (Dietler and Herbich, 1998; Lemonnier, 1986; Sillar and Tite, 2000). Thus, many discourses on technology have turned from evolutionary and functionalist perspectives to other trends that argue that technology is related to a certain savoir faire and becomes the social dimension of the techniques. We have already seen how the creation of any piece of pottery involves a characteristic sequence of technological choices. For instance, potters select certain clays and tempers and prepare the paste using precise proportions as well as following specific sequences and procedures. All these choices and the creation of a given ceramic object are the result of a series of interactions between people and one or more technologies, affecting the processes of pottery manufacture, use, maintenance and deposition. Hence, the concept of technology is understood in a broad sense covering from the selection of the raw materials and manufacturing techniques to the multiple functions and meanings related to the objects (Schiffer, 2004).

In many cases, this social dimension of technology is not explicitly stated by researchers. This aspect is minimised and disguised in contemporary Western societies where, in contrast, it is quite common to emphasise the close relation existing between material and technical factors as well as the knowledge necessary to produce them. However, technology involves issues that go beyond the application of certain techniques, materials and knowledge. These parameters represent the instrumental basis for technology, but this concept clearly relates to practices and human actions developed in specific social contexts (Creswell, 2003). In a broad sense, technology is related to the particular way people do things (Sigaut, 1994). Therefore, technology is not just a corpus of knowledge, an array of materials and a number of techniques which creates materiality to meet given needs. Technology, and therefore techniques and chaînes opératoires, represents a means by which human beings, either consciously or unconsciously, attempt to address purposes that go beyond materiality itself. But, what is the connection between technology, chaînes opératoires and techniques? What kind of useful information do these concepts provide to the archaeological research?

The Anthropology of Techniques (Lemonnier, 1986, 1993; Sigaut, 1994) considers that certain chaînes opératoires are representative and characteristic of a particular society. The continuous repetition of technical gestures and sequences in time and space favours the stabilization of specific ways of savoir faire. This concept includes the sequential and operational knowledge resulting from specific mental representations that is necessary to produce artefacts (Vidal and García Rosselló, 2009). Therefore, technology is always associated with some knowledge, which may be conscious and discursive or not, that allows establishing a relationship between specific chaînes opératoires and their final products. In fact, as pointed out by Dobres and Hoffman (1994), in ancient Greek the term tekhne refers to the technical knowledge and skills 
of the individuals and not with the materiality itself. The know-how or savoir faire transmitted between individuals is essential not only to understand how ceramics are produced but their entire life cycle. In this way, techniques and technology conceptually unify knowledge, practice and experience. Recording the continuities and ruptures present in the technological choices that characterise the different phases of the chaîne opératoire can also be approached changes related to knowledge and the characteristic rationale of each society.

As stated before, there is a close connection between the artefact manufactured and the production process put in practice. In this sense, the use of different actions, techniques and materials may condition the achievement of completely different pottery vessels, providing individuals with a mean to articulate their actions. Through pottery characterization we can associate certain traits in the materials with specific technical actions and eventually with sequences of particular technical gestures that make up a chaîne opératoire and a particular technological tradition. Ultimately, the technological tradition synthesizes a particular know-how and way of expression through material culture. The concept of technological tradition makes sense when we consider that no object lasts forever, thus the end of the life cycle of a given ceramic object takes place when it loses its physical and/or intangible properties. At this point, the pottery has no longer a function in society and can be replaced by a similar artefact. This replacement process enables and encourages the development of specific technological traditions which would create vessels manufactured in the same way and having the same characteristic as the later pottery.

Furthermore, as mentioned earlier, each technological choice made during the production process has an effect on one or more formal properties of the final product. Hence, the technical actions undertaken will also influence the several phases involved in the manufacturing, use and maintenance processes. The importance of approaching all the phases involved in the chaine opératoire lies in the fact that the potters' actions are carried out in relation to the production process as a whole. This assumption affects both the physical and the ideal factors involved in the manufacturing of ceramics. On the one hand, each technique and material used in the chaîne opératoire is embedded with social and symbolic meaning. Moreover, the chaîne opératoire must not be seen as a simple addition of these meanings; it rather involves complex connections and movements back and forth among all of them. The use of a particular technical gesture affects the meaning of the following and the complete set determines the overall meaning of the pottery production process and the object itself (Dobres, 2000).

On the other hand, we have to consider the physical constraints that the use of certain materials and techniques has in the different phases of the chaîne operatoire. Thus, materials and techniques can also limit the potters' freewill regarding the use of new technological choices. In short, all the techniques, materials and agents directly or indirectly involved in the production are closely related, so that changes in the materials and/or techniques used in a specific stage induce changes in the other 
(Sillar and Tite, 2000). For example, the addition of temper may increase the difficulty of building of thinner walls while it generates a porous microstructure in the matrix which in turn has effects in the firing process and the final properties of the vessel.

This interrelationship has a dual effect, since some phases of the production process can be maximised while other are minimised. This combination of complex causal processes between different technological choices involving materials and techniques is one of the aspects that make possible the existence of technologies. Each technology involves a complex interaction of actions and phenomena that takes place during the several stages of manufacture, use and maintenance of the material culture. Thus, each potter's decision potentially involves a series of compromises in which some groups of actions can be performed at the expense of another. In the same line of thought, a particular technology also interacts with other technologies enabling us, in long-term research studies, to address issues that go beyond the ceramic record itself (Balfet, 1984; Gibson and Woods, 1990; Van As, 1984).

So far we have been briefly discussed the technology concept and its connection with certain sequences of actions involving various techniques and materials. We have also evaluated the role of the chaînes opératoires and some related elements in the study of past societies and their material culture. We will later discuss in depth this question in connection with the existence of specific technological traditions and their long duration in certain geographical areas. In short, the characterization of chaines opératoires based on materials and techniques is an adequate procedure to establish the existence of certain technological traditions. These traditions are closely related to particular social, functional and environmental contexts in which knowledge is transmitted. It is within these complex contexts in which the chaines opératoires have to be understood and interpreted. The study of ceramic technology only makes sense when we carry out social explanations that move back and forth from the materiality to the individual and their actions.

Nevertheless, before facing this major interpretative level, it is convenient to overview first the technical processes that potters usually carry out in each phase of the chaîne opératoire. What is more important, we have to reflect on the way these phases can be addressed by means of the analysis of the pottery sherds. In this reflection we must also pay special attention to the technological implications that the use of certain materials and techniques have in the development of other phases of the production process. The actions and choices that the potters introduce during the manufacturing process cause textural, mineralogical and physicochemical changes in the raw materials that affect their properties and the type of final product achieved. Thus, we can summarize in the chaîne opératoire all the actions performed by the artisans involving changes in the clay during the different phases of pottery production, from raw materials management to the rest of the operations undertaken to manufacture the pottery vessel. 
While several stages compose the pottery chaîne opératoire (see, for instance, Calvo et al., 2004a; García Rosselló and Calvo, 2013), the phases most commonly discussed in ceramic paste studies are:

1. Clay selection and extraction

2. Paste preparation

3. Modelling

4. Drying

5. Surface treatment

6. Firing

There are many handbooks (e.g., Cuomo di Caprio, 1985; Gibson and Woods, 1990; Morales, 2005; Orton et al., 1993; Rice, 1987; Rye 1981; Shepard, 1971; Sinopoli, 1991; Velde and Druc, 1999) that provide a comprehensive approach to all the phases of the chaîne opératoire in pottery production. The aim here is to make a brief overview of the different technological options that potters have in each phase of the manufacturing process and the way they can be identified through the analysis of the archaeological record. Thus, in order to avoid repeating what was already mentioned in these volumes we will only emphasise and clarify those aspects that are considered most relevant. Furthermore, to best undertake the identification of the techniques and raw materials through the ceramic traits we have used a framework enhanced with ethnoarchaeological and experimental studies. As discussed at the end of this chapter, both disciplines provide a broader perspective regarding the technological choices that can be potentially used to produce traditional pottery and their technical, social and symbolic significance.

Finally, it should be highlighted that from a materialistic viewpoint the concept of chaîne opératoire also characterises the mutability of the matter, from the raw clay to the fired product. Therefore, through the analysis of each manufacturing phase we can also approach the complex physicochemical changes undergoing the materials along the production process. In this sense, it will be also emphasised which procedures and factors cause marked physicochemical differences between ceramics and the role of the human actions in these processes of change. 